\title{
La regulación del Espacio Audiovisual Ampliado en Argentina de 2007-2015
}

\author{
Audiovisual Space Regulation expanded in Argentina, 2007-2015
}

\section{Regulação do Espaço Audiovisual expandida na Argentina de 2007-2015}

\section{Santiago Marino}

Doutor em Ciências Sociais, Comunicação e Cultura. Mestre e Bacharel em Ciências da Comunicação (FSOC-UBA). Diretor do Mestrado em "Indústrias Culturais: política e gestão" da Universidade Nacional de Quilmes (UNQ).

<sgomarino@gmail.com>

\section{RESUMEN}

Este artículo tiene como objetivo presentar un análisis descriptivo de las regulaciones de cada uno de los sectores que operan en el espacio audiovisual ampliado (EAA). El objeto que se analiza es el conjunto de normativas sancionadas y/o reformadas para este espacio en Argentina durante los dos mandatos presidenciales de Cristina Fernández de Kirchner. Se concibe las decisiones aplicadas en materia regulatoria, como instrumentos centrales que materializan las políticas de comunicación. Se espera que este recorrido permita describir las dinámicas del objeto, siga las pautas de análisis establecidas e identifique las formas y prácticas de regulación divergentes para un espacio que tiende a converger.

Palabras clave: Regulación del Espacio Audiovisual. Políticas de comunicación. Cine y televisión.

\section{ABSTRACT}

This article's goal is to present a descriptive analysis of the regulations to each of the sectors that operate in the augmented audiovisual space (AAS). The objects of study are the revised and/or amended principles for such space in Argentina, during both Cristina Fernández de Kirchner's presidential mandates. Decisions are applied in terms of regulation, central instruments that incorporate communication policies. We hope the route to describe such dynamics will follow the analysis directions and detect practical forms of divergent regulation for a space that tends to convert.

Keywords: Audiovisual Space Regulation. Communication policies. Cinema and television.

\section{RESUMO}

Este artigo tem como objetivo apresentar uma análise descritiva dos regulamentos para cada um dos setores que operam no espaço audiovisual ampliado (EAA). $O$ objeto a ser analisado é o conjunto de normas editadas e/ ou reformadas para este espaço na Argentina, durante os dois mandatos presidenciais de Cristina Fernández de Kirchner. É concebido decisões aplicadas em matéria de regulação, como instrumentos centrais que incorporem políticas de comunicação. Esperase que o percurso para descrever a dinâmica do objeto, siga as orientações da análise estabelecida e identifique formas práticas de regulação divergente para um espaço que tende a convergir.

Palavras-chave: Regulação do espaço audiovisual. Políticas de Comunicação. Cinema e televisão

\section{Introducción}

El presente trabajo tiene como objetivo presentar un análisis descriptivo de las regulaciones para cada uno de los sectores que operan en el espacio audiovisual ampliado (EAA). El objeto que se analiza es el conjunto de normativas 
sancionadas y/o reformadas para este espacio en Argentina durante los dos mandatos presidenciales de Cristina Fernández de Kirchner. Se concibe a las decisiones aplicadas en materia regulatoria como instrumentos centrales que materializan las políticas de comunicación.

Se espera que el recorrido permita describir las dinámicas del objeto, seguir las pautas de análisis establecidas e identificar las formas y prácticas de regulación divergentes para un espacio que tiende a converger.

La posibilidad de pensar en la configuración del EAA es comprendido aquí a partir del surgimiento y expansión de tecnologías que rompen inercias y formas de organización productivas y de consumo en las distintas industrias culturales (Marino, 2015). Es integrado por los sectores del Cine, la Televisión (abierta y de pago, analógica y digital) y los servicios de distribución de contenidos audiovisuales online. La incorporación y combinación de nuevas formas de producción y circulación (Monzonzillo, 2011) son fruto de un desarrollo no espontáneo, que obedece tanto a la evolución de las Tecnologías de Información y Comunicación (TICs) vinculadas a las industrias audiovisuales como a factores externos y estructurales del capital globalizado (Martell, 2011).

El recorrido del texto propone, en primer lugar, la presentación de los aspectos teóricos y metodológicos que encuadran la investigación y las premisas de las que parte. A continuación se detallan los sectores que integran el EAA dentro de las industrias culturales. Más adelante se describen las regulaciones adoptadas en el recorte temporal establecido, ya sean sanciones de leyes integrales, reformas o decretos y resoluciones. Para finalizar, se especifica integralmente el plexo normativo resultante y se lo evalúa como marco encuadra (entre otros aspectos) el desarrollo por venir.

\section{Aspectos teóricos y metodológicos}

La línea teórica que guía el abordaje es la de las políticas públicas de comunicación, comprendidas como un conjunto articulado de normas y reglas y la aplicación de planes, programas y proyectos en un período histórico dado (Van Cuilemburg y Mc Quail, 2003). La investigación parte de concebir la centralidad de las normas como materialización de la intervención estatal en el sector, fruto de la interacción con los diferentes actores involucrados (Fredman, 2006). Se entiende que las políticas no se agotan en la sanción de una o varias normativas que reemplacen a las anteriores, ni en la reforma de algunos aspectos de las leyes vigentes. Sino que se desagregan luego en acciones de gestión e 
implementación. Pero se subraya que el objeto de este trabajo está compuesto por las medidas regulatorias aplicadas entre 2007 y 2015.

Como aspecto clave del contexto estudiado, la convergencia será entendida como el proceso tecnológico, económico y social que posibilita que los sistemas de Telecomunicaciones (transmisión de datos, telefonía e internet) y el audiovisual (distribución de contenidos mediante un vínculo que puede ser el aire, cable o satelital, gratuito o de pago) convivan en una misma red y habiliten combinaciones de usos, formas de producción, distribución, financiamiento y consumos (Becerra, 2003, 2015). Este estudio indagará en los modos en que la nueva regulación contempla dicho desarrollo.

Por su parte, la concentración de la propiedad del sistema de medios es definida como un proceso complejo e integral, inmanente a las industrias culturales y que debe ser analizado desde distintos aspectos, a saber: geográfico, económico, sociopolítico y cultural (Mastrini y Becerra, 2006). Se centra en el incremento de la eficiencia o el crecimiento del poder de mercado por parte de uno o varios jugadores.

El recorrido metodológico implicará dar cuenta del proceso de elaboración de las regulaciones que materializan las políticas, de su contexto y de los aspectos que su diseño afectan (temáticas reguladas, organismo de aplicación, diseños institucionales, encuadres para las instancias de producción y distribución de contenidos, actores). A esto se agregará la sistematización de principales rasgos de la implementación. Dicha descripción se combinará con entrevistas realizadas a actores involucrados.

Los sectores: el complejo espacio audiovisual en las industrias culturales

\section{El Cine}

El sector cinematográfico posee una peculiaridad en Argentina: cuenta con una política pública de largo plazo que establece mecanismos de estímulo y protección a la producción local y ha sido sostenida más allá de cambios políticos yeconómicos (Marino, 2014a). Su legitimidad es amplia y su permanencia parece indiscutible, a pesar de lo desparejo de sus resultados, del paso del tiempo y de la necesidad de repensar algunas de sus líneas, con el objetivo de optimizar el uso de sus recursos y ampliar las condiciones de acceso de los ciudadanos a este tipo de contenidos, fundamentales en la construcción de identidades culturales nacionales. 
La permanencia en el tiempo dela política de cineen nuestro país consolidó un sistema con sus rasgos positivos - como el fomento a la producción y (en menor medida) a la exhibición - y limitaciones notables (la concentración y extranjerización de la propiedad en las instancias de producción y distribución, la escasez de pantallas, la falta de control sobre el cumplimiento de cuotas de pantalla nacional' ${ }^{1}$ y media de continuidad ${ }^{2}$ ) que aún no pueden resolverse (Marino, 2014a).

El cine es un medio peculiar tanto por sus rasgos económicos como por su rol en la construcción de relatos que aportan a la constitución de identidades (Getino, 2005). En países como la Argentina requiere de políticas públicas que garanticen su existencia, dada su estructura de costos, el tamaño del mercado y el dominio de las producciones extranjeras. La industria local es aparentemente débil pero compone, a su vez, la segunda en idioma español y es una de las diez más importantes de occidente 3 . En este esquema, unos pocos films locales resultan "elegidos" por el público, superan la cantidad necesaria de espectadores para cubrir los costos de producción y convertirse en éxitos de taquilla (unos 700 mil tickets) y de alguna manera hacer posible la generación del resto (Perelman y Seivach, 2003).

\section{La regulación y los cambios}

La actividad estaba originalmente regulada por la Ley $17.741^{4}$ y fue reformada mediante las leyes 20.170/73, 21.505/77, 24.377/94 y el decreto $1.536 / 02^{5}$. La norma (con sucesivas reformas y reglamentaciones) establece las condiciones de funcionamiento y fomenta la actividad, crea el Instituto Nacional de Cine y Artes Audiovisuales (INCAA) como organismo de aplicación; define lo que entiende por "película nacional", establece las condiciones para su comercialización y exhibición obligatoria, genera y destina recursos para el crédito y el subsidio a la producción, fomenta las coproducciones, promociona el cine nacional en el exterior y prevé el apoyo y protección al cortometraje.

1 Es la obligación que tienen los exhibidores de incluir en su programación determinada cantidad de películas nacionales en su cronograma de estrenos.

2 Es la obligación que tienen los exhibidores de incluir en su programación determinada cantidad de películas nacionales en su cronograma de estrenos.

3 Luego de España. Véase además: Rovito (2010). “El nuevo paradigma del negocio cinematográfico" (p. 105-112). En: Valor y símbolo, dos siglos de industrias culturales en la Argentina. Buenos Aires: Secretaría de Cultura. Sistema de Información Cultural de la Argentina (SINCA).

4 Boletín oficial, 30 de mayo de 1968.

5 Que recuperó la autarquía para el INCAA luego del estallido de la crisis de diciembre de 2001. 
El nuevo escenario derivó en un crecimiento importante de la cantidad de películas producidas y estrenadas, como así también en el reconocimiento de la obra de directores argentinos. Pero no pudo solucionar dilemas estructurales, como la falta de control para la aplicación de las mejores medidas y las dificultades en las condiciones de estreno y exhibición.

Durante el período estudiado en este trabajo (2007-2015), este aspecto se resolvió - en términos de Oszlak y O'Donnell (1984) con un agregado a la política del sector. Desde enero de 2012 el INCAA reorganizó las condiciones de los lanzamientos de las películas mediante las siguientes medidas definidas en resoluciones del organismo:

- creación de un calendario trimestral de estrenos nacionales;

- reclasificación de las salas en "circuito ampliado" (hasta tres pantallas) y "circuito multipantallas" (cuatro pantallas o más);

- fondos de apoyo para gastos de distribución y promoción ${ }^{6}$ de los films que se estrenan en los espacios con pocas salas (Marino, 2014a) ${ }^{7}$.

Por su parte, como en otros momentos de la historia del país, la regulación del sector de la radiodifusión implicó novedades para el Cine. Así, el modo en que la Ley de Servicios de Comunicación Audiovisual en 2009 interpeló al cine resultó positivo en el marco regulatorio, aunque no se constate en la implementación. Tal como sostiene Sahores:

\begin{abstract}
Desde la sanción de la LSCA hasta finales de 2014, ninguno de los canales presentó la documentación que acredite la compra de los derechos de emisión de películas ni, menos que menos, cumplió con la exhibición de esos estrenos en la pantalla. Según datos aportados por el Sindicato de Cine, en los primeros nueve meses de 2012, entre América (cero), Canal 9 (una), Telefé (ocho) y Canal 13 (dos) emitieron apenas once películas argentinas. Entre ellas, una sola inició su rodaje antes de la promulgación de la ley. Se trata del documental sobre Malvinas, 14 de junio, que emitió Canal 9. Hasta entonces tienen datos sistematizados (Sahores, 2014) ${ }^{8}$.
\end{abstract}

Respecto de la instancia de distribución, durante el año 2013 y luego de una serie de reuniones entre el - por entonces - Secretario de Comercio Guillermo Moreno y los ejecutivos de las filiales locales de las cinco majors más poderosas que operan en la Argentina (Sony, Disney, UIP, Warner y Fox), se

6 Un monto de $\$ 30.000$.

7 Para profundizar este aspecto y constatar datos puede ampliarse en Marino (2014a).

8 Entrevista realizada con el periodista especializado Esteban Sahores, Buenos Aires, mayo de 2014. 
constata un cambio en el rol de estas empresas y la distribución y exhibición de los films argentinos. Estos capitales extranjeros generaron nuevas alianzas con las principales productoras locales, a partir de lo cual los films generados por la "crema" de lo industria local (apoyados en el star system de directores, como Juan José Campanella, o de actores, como Ricardo Darín y Guillermo Francella) comenzaron a ser comercializadas por las grandes distribuidoras. El resultado fue importante en términos de mejores condiciones de estreno y taquilla (Marino, 2014a), pero no en términos de desarrollo de la industria local, dado que por un lado, son unos pocos productos argentinos los que acceden a dicha condición, mientras que las distribuidoras locales históricamente abocadas a trabajar con material argentino comenzaron a perder terreno. Es necesario destacar además que esta medida no se expresa en resolución específica alguna.

Contodo,loscambiosimplementadosenelmarcoregulatoriodelaactividad cinematográfica entre 2007 y 2015 fueron pocos, focalizados y no generados por acciones del propio sector, sino por regulaciones de otras áreas del EAA.

\section{La Ley Audiovisual}

Dentro del sistema de radiodifusión se ubican la radio (AM y FM) y la Televisión (abierta y de pago, analógica y digital). Durante el período estudiado en este trabajo en Argentina se tomaron decisiones en materia regulatoria para la Televisión en términos generales, por un lado, y para su proceso de digitalización, por otro. En ese contexto se describe, en primera lugar, la ley de Servicios de Comunicación Audiovisual (LSCA).

El Congreso sancionó la LSCA 26522/09 en medio de aquel conflicto. Ahora bien, la norma fue elaborada con apoyo masivo de varios sectores, entre los que se destaca la acción de la Coalición por una Radiodifusión Democrática?. Uno de los aspectos novedosos lo constituye la reserva del 33\% de todo el espectro para el sector privado no comercial. La ley reconoce tres tipos de prestadores: estatales, privados comerciales, y privados no comerciales. En relación con el diseño institucional, creó la Autoridad Federal de Servicios de Comunicación Audiovisual (AFSCA) compuesta por siete miembros, que son designados por el Poder Ejecutivo Nacional (PEN) (dos), el Congreso (tres, de las primeras minorías) y el Consejo Federal de Comunicación Audiovisual (COFECA, tres). Precisamente otro elemento a destacar es la conformación del COFECA, organismo de discusión de Políticas en el que participan representantes de

9 Para indagar sobre su composición y objetivos se recomienda ver más en Marino (2014b). 
las provincias, las empresas, los radiodifusores sin fines de lucro, los pueblos originarios, los medios públicos, los sindicatos de trabajadores de medios y las universidades nacionales. Se creó además la Defensoría del Público y el Consejo Asesor del Audiovisual y la Infancia.

Otro capítulo central es el que procura impedir la concentración de los medios. Limita a diez el máximo de licencias de radio y TV abierta que puede disponer una misma empresa o persona, 24 para los operadores de TV por Cable y una licencia nacional para la distribución por satélite. A la vez, se permite a las distribuidoras de cable sólo incluir una señal propia. Para todos se establece además el tope de $35 \%$ del mercado potencial. Se prohíbe también la propiedad cruzada (abierta y de pago) en la misma zona de cobertura, mas no la de medios impresos y electrónicos. En materia de contenidos fija altas cuotas de producción nacional (60\%), de producción propia (30\%), e independiente (10\%) de acuerdo a la distribución de cobertura, como así también la exigencia de exhibir films de estrenos nacionales en TV abierta. La norma vincula el sistema de radiodifusión con otras industrias culturales, pues parte de los gravámenes que deben abonar los radiodifusores por el uso del espectro, es girado a la industria del cine y a la de la música ${ }^{10}$.

El debate y los giros continuaron una vez aprobada la ley. Su aplicación resulta sesgada, por varias razones, estrategias y actores. En el período pueden reconocerse tres etapas:

- por causas externas: desde noviembre de 2009 hasta diciembre de 2011;

- por causas combinadas: desde diciembre de 2011 - a partir del comienzo del segundo mandato de Fernández de Kirchner - y hasta octubre de 2013, por el fallo de la Corte Suprema de Justicia de la Nación respecto del proceso judicial iniciado por el Grupo Clarín;

- Adecuación y aplicación sesgada: desde aquella decisión judicial en adelante (Marino, 2014b).

Luego de un proceso judicial muy complejo, la LSCA es todavía parte de un debate intenso. Y resulta un desafío para pensar los medios en el futuro, cuyas tendencias centrales son la concentración (que limita pero cuyos aspectos no se aplican) y la convergencia, que quedó fuera de su marco.

10 Este y otros aspectos centrales del proceso fueron trabajados profundamente en investigaciones anteriores. Ver más en Marino (2014b y 2015). 


\section{La Televisión Digital}

El despliegue de la televisión digital en Argentina resulta una experiencia singular y llena de contradicciones. Específicamente en lo que refiere al desarrollo de la Televisión Digital Terrestre (TDT), que es parte de la radiodifusión y viene a reemplazar a la analógica hertziana. Se suma al conjunto de políticas que implementa el Estado en este período para el EAA y materializa decisiones regulatorias propias. Configura un rol central e inédito de lo público en todas las instancias, mientras que el sector privado comercial observa y sigue su camino en el mercado de pago.

El Estado argentino asumió el liderazgo para el desarrollo de la TDT. En esa dirección, a través del Decreto 1148/09 se creó el Sistema Argentino de Televisión Digital Terrestre (STDA). Conformó el Consejo Asesor del STDA en la órbita del Ministerio de Planificación Federal, Inversión Pública y Servicios (MINPLAN). El decreto estableció un plazo de diez años para realizar el proceso de transición digital, por lo cual 2019 es la fecha para el apagón analógico en nuestro país.

Desde entonces el Gobierno mantuvo una fuerte iniciativa política para desarrollar la TDT, que incluyó la instalación de antenas en numerosas ciudades del país, la entrega gratuita de Set Top Box para adaptar los televisores analógicos, la creación de fondo para la producción de contenidos, y un programa para financiar la compra de televisores con sintonizador digital. Casi simultáneamente a la adopción de la norma se sancionó la LSCA, que no aborda de manera exhaustiva el problema de la convergencia y de la transición al entorno digital. Debe marcarse que el artículo 88 de la LSCA establece que la AFSCA confeccionará, junto a la autoridad técnica pertinente, la norma nacional del servicio de TDT y administrará la transición analógico-digital (Krakowiak y otros, 2012).

Las acciones del Estado para el despliegue de la televisión digital se toparon con un contexto adverso debido a la altísima penetración de la televisión por cable (aproximadamente el $90 \%$ de los hogares). La oferta de los sistemas de cable (60 o más canales, que incluyen deportes, películas y series) se han mostrado por ahora más atractivos que la televisión digital que oferta 30 canales, incluso aunque esta última sea gratuita (Mastrini y otros, 2014).

La adopción del estándar ISDB-Tb (Servicios Integrados de Difusión Digital Terrestre) se presentó durante la cumbre de la Unión de Naciones Sudamericanas (UNASUR) celebrado en la ciudad de Bariloche en 2009. La Secretaria de Comunicaciones (dependiente del PEN) aprobó la Resolución 04/06 que estableció la creación de la Comisión de Estudio y Análisis de los Sistemas de 
Televisión Digital. Dicha acción marcó un perfil técnico productivo a las políticas para el desarrollo de la televisión digital, que luego se llevarían a cabo bajo el liderazgo del MINPLAN. La plataforma abierta o hertziana es el último sistema de televisión en iniciar la migración tecnológica. El estado argentino ha asumido un rol protagonista para llevar adelante el despliegue de la televisión digital abierta (Krakowiak y otros, 2012).

El sistema denominado desde entonces de TDA $^{11}$ (terrestre y satelital) se erige sobre la plataforma diseñada por la empresa ARSAT S.A. La infraestructura tecnológica permite la multiplicación de las señales abiertas de televisión y su transmisión a través de las plantas transmisoras. Estas se han instalado en las ciudades del país con mayor densidad poblacional y/o ubicación geográfica estratégica, a partir de un cronograma por etapas. Durante el periodo 20102012 se alcanzó una cobertura del 75\% de la población a través de 51 estaciones digitales terrestres en funcionamiento, más 90 localidades rurales que reciben Televisión digital por satélite (Mastrini y otros, 2014). Esa cobertura llega al 85\% a finales de $2015^{12}$.

Con el objetivo de facilitar el acceso a la nueva tecnología, se puso en marcha el Plan Operativo de Acceso a“MiTV Digital", el cual implica la distribución gratuita de los decodificadores a los sectores más vulnerables de la población. Por otra parte, el sistema de TDA se articula con programas que promueven la producción de contenidos con énfasis en la promoción del federalismo. En este sentido, se aprobó el decreto 1528/12 que considera a la actividad desarrollada por los productores de contenidos audiovisuales de capitales públicos, privados o mixtos como una actividad productiva asimilable a la industrial, de manera que pueda gozar de los beneficios y exenciones tributarias. En sintonía con la LSCA, creó el Programa Polos Audiovisuales Tecnológicos, que divide al país en nueve regiones o polos, junto con las universidades nacionales que organizan a los actores del sector audiovisual televisivo a través de nodos para la producción de contenidos para Televisión digital.

\section{Argentina Digital}

La importancia de la exclusión de las empresas de Telecomunicaciones de la posibilidad de brindar "servicios de radiodifusión por suscripción prestados por enlace físico" (básicamente, cable), tuvo como efecto desconocer

11 Por “Televisión Digital Abierta”, que se relaciona con la que usa espectro, y también por "Televisión Digital Argentina".

12 Fuente: <http://www.tda.gob.ar/> última visita 4/11/2015. 
la convergencia tecnológica, que es inexorable. Como sostiene el investigador Fontanals

\begin{abstract}
la crítica al proyecto original de la ley desconocía las fuertes limitaciones particulares que el anteproyecto incluía para que las empresas de telecomunicaciones pudieran participar (y que se sumaban a las generales para todos los prestadores) tales como: a) la existencia de competencia efectiva en la localidad respectiva (la presencia de otro prestador y un dictamen de la Comisión Nacional de Defensa de la Competencia); b) la exigencia de libre interconexión de sus redes y la desagregación de su infraestructura (facilitando el acceso a postes, mástiles y ductos a terceros que quisieran proveer el servicio); c) la vigencia de la portabilidad telefónica (fija y móvil) y d) la obligación de conformar unidades de negocio separadas con el fin de evitar el uso de subsidios cruzados entre servicios. En un escenario así se condicionaba la incursión de las telecomunicaciones en la TV por cable, y seguramente se concentraría en las localidades más rentables, donde competiría con los cableoperadores más consolidados, como Cablevisión, de Clarín, o Supercanal, de VilaManzano (Fontanals, 2014) ${ }^{13}$.
\end{abstract}

Pero tras cinco años de sancionada la LSCA el gobierno de Cristina Fernández de Kirchner sancionó una nueva ley que interpela a sectores del EAA. Y completó así un póker de regulación.

En noviembre de 2014 el PEN presentó el proyecto denominado“Argentina Digital", traía numerosos peligros y desafíos. Podría ser un aporte para mejorar el plexo normativo, diseñar y aplicar una serie de políticas convergentes para el sistema. La medida respondió a una iniciativa política que podrá tener impactos económicos, regulatorios y socioculturales.

La Ley 27078 de Tecnologías de la Información y las Comunicaciones "Argentina Digital" se aprobó el 16 de diciembre de 2014. Entre los aspectos centrales se destacan la declaración de "servicio público" para el uso y acceso a las redes de Telecomunicaciones para licenciatarios de servicios de TICs (lo que permite la regulación de tarifas en el mercado mayorista); la eliminación de la larga distancia nacional y la obligación de provisión de velocidades mínimas de conexiones a internet. Mientras que no amplió la definición de "servicio público" para la telefonía móvil, el mercado más utilizado por los usuarios en Argentina (Perea y Retegui, 2012).

13 Entrevista realizada con Gustavo Fontanals por correo electrónico, julio de 2014. 
En relación al EAA, la ley autoriza a las empresas de telefonía fija a brindar el servicio de Televisión por suscripción ${ }^{14}$, lo que implica un cambio drástico respecto de lo regulado en 2009 con la LSCA, pues transforma las condiciones de regulación y permite expandir el negocio a las empresas de Telecomunicaciones, impedidas hasta entonces de operar en ese mercado. Tal como entienden los investigadores Mastrini y Becerra

\begin{abstract}
La mayor novedad de este marco regulatorio es que permite el acceso de las empresas telefónicas a brindar servicios de comunicación audiovisual. [...] Junto con este nuevo encuadre que favorece la creación de un mercado convergente, se aprobó la formación de una nueva autoridad regulatoria, se estableció la neutralidad de la red, y se establecieron condiciones de servicio y de competencia para los licenciatarios del sector. El objeto de regulación de la nueva ley es la infraestructura de transporte de comunicaciones. Abarca las redes telefónicas, de conexiones de banda ancha y de televisión por cable. El proyecto no regula contenidos y dispone la "neutralidad de la red", es decir, la no discriminación de ningún tipo de contenidos (Mastrini y Becerra, 2015).
\end{abstract}

Esta posibilidad de que las operadoras de Telecomunicaciones puedan prestar servicios audiovisuales es la novedad central. Aunque en realidad algunas de esas empresas ya están presentes en el mercado de la Televisión abierta ${ }^{15}$. Su capacidad económica - a partir del tamaño del mercado concentrado y de escala amplia en el que operan - podría redundar en condiciones de competencia desigual con los agentes originarios del mercado de medios tradicionales. En esa línea, el rol del Estado en su capacidad de controlar operaciones cruzadas y niveles de competencia será fundamental, para evitar que las grandes empresas de Telecomunicaciones los fagociten. La línea parece habilitar un nuevo tipo de concentración: la convergente (Becerra, 2015).

Si se compara con el proceso que derivó en la sanción de la LSCA, la discusión sobre los contenidos de "Argentina Digital" fue acotada y reducida en plazos, formas y tiempos. Aunque permitió optimizar cuestiones centrales que el proyecto no resolvía. De todos modos, el rasgo central de la nueva

14 A excepción del sistema satelital, según indica el artículo 9.

15 Como es el caso de Telefónica, quien controla con empresas subsidiarias Canal 11 Telefé. Ver más en Di Santi y Riera (mimeo, 2015) y Becerra (2014). 
norma radica en la discrecionalidad habilitada para el PEN, que deberá definir cuestiones centrales en el Decreto Reglamentario.

\begin{abstract}
Los cuatro puntos centrales sobre los que el texto de la ley no establece definiciones claras son: capacidades de la autoridad de aplicación y control; definición de tipo de servicio (entre lo que se incluye como servicio público - interoperabilidad de las redes y la telefonía fija - y lo que se excluye - elefonía móvil-); reglas de competencia y habilitación de cruces entre telefonía y audiovisual; neutralidad de la red (que se define de modo escueto y no integral) (Mastrini y Becerra 2015).
\end{abstract}

A través del Decreto 677/2015 publicado en el Boletín Oficial del 29 de abril de 2015, se puso en funcionamiento Autoridad Federal de Tecnologías de Información y Comunicación (AFTIC). Según lo establecido en la ley Argentina Digital, este nuevo organismo continúa con las atribuciones que tenía la Secretaría de Comunicaciones y la Comisión Nacional de Comunicaciones, además de incorporar bajo su órbita el plan Nacional Argentina Conectada y las empresas ARSAT y Correo Argentino. Al igual que la AFSCA, su directorio contará con siete miembros, designados con similar carácter representativo: dos por el PEN; tres por la Comisión Bicameral de Promoción y Seguimiento de la Comunicación Audiovisual, las Tecnologías de las Telecomunicaciones y la Digitalización, correspondientes al Poder Legislativo; un representante de las Provincias y la CABA y uno designado por el Consejo Federal de las Tecnologías de las Telecomunicaciones y la Digitalización, conformado por todos los actores del ecosistema de las TIC.

Ya encuadrado en esta norma, en setiembre de 2015 el Grupo Clarín anunció la compra de la empresa Nextel, el cuarto operador del mercado de telefonía móvil y transmisión de paquetes de datos de nuestro país. La información se difundió a partir de la obligación del Grupo por ser un operador de la Bolsa de Valores de Argentina e Inglaterra. Inmediatamente se generó una (nueva) polémica con el Gobierno. Y la AFTIC resolvió no autorizar la transacción. Esta decisión se complementa con la negativa que el organismo definió al pedido del empresario estadounidense David Martínez (titular del Fondo Fintech y socio del Grupo Clarín en la empresa Cablevisión) para comprar la empresa Telecom. Estos acontecimientos avizoran nuevos episodios para la novela Avenida Clarín (Marino, 2014b). Permiten sostener el axioma según el cual las cuestiones de las cuestiones de la disputa política inciden tan significativamente en el diseño y 
aplicación de políticas públicas en Argentina. Y habilitan interrogantes en torno al futuro del mercado convergente.

\section{Lo no regulado}

Así como resulta clara la diferencia entre Televisión abierta (usa el éter para transmitir, es de recepción gratuita) y de Pago (vía cable o satélite), en la actualidad hablamos de la digital, destacada por sus ventajas (calidad de imagen y sonido, optimización de uso del espectro, interacción con el usuario, multiplicación de la oferta) y por sus incertidumbres (segmentación de audiencias y mercados, inversión para producir y acceder, modelos de financiamiento). Al mismo tiempo se instala, un conjunto de nuevos actores (los desarrolladores de la TVOTT) que pueden insertarse en el mercado con ventajas comparativas respecto de los actores del mercado incumbente (Páez, 2014). La escala a desarrollar es global porque son jugadores trasnacionales (como el caso de Netflix, HBO, Fox, entre otros); operan en una red que no es propia ni tienen que desarrollar $y / o$ mantener (para este ejemplo sirven igualmente los casos anteriores); y algunos son operadores de la red que agregan valor por prestar el servicio de conexión además de ofrecer contenidos audiovisuales (es el caso de Telefónica, con Speedy y "On video" y de Cablevisión, con Fibertel y el proyecto de desarrollar un OTT propio en 2016).

El mercado televisivo desanda este camino en dos vías. Por un lado, un segmento que se suma a la Televisión de alta definición (HD) y a las nuevas formas de a partir de la expansión comercial y la capacidad de pago de los consumidores. Se ha acelerado la incorporación hogareña de equipamiento, pero no se comprueba aun la expansión en el consumo ${ }^{16}$ ni el ofrecimiento del servicio (sólo los grandes operadores han invertido en su red y pueden ofrecerlo). Mientras el Estado regula de modo diversificado en el período estudiado, desconoce las operaciones existentes y/o fracasa en el intento de que al menos tributen de algún modo ${ }^{17}$.

16 Poco más del 25\% de los abonados paga el plus por el servicio, según fuentes de las propias empresas, Cablevisión y DirecTV.

17 Como el intento de la Agencia Tributaria del Gobierno de CABA de cobrar el impuesto a los Ingresos Brutos a los prestadores de todo tipo de servicios OTT 


\section{Legislación (y política) comparada}

Argentina presenta cuatro regulaciones que materializan líneas de políticas públicas para el conjunto de sectores que tienden a integrase en el EAA dentro de las industrias culturales. La normativa parece trazar caminos paralelos en un espacio en el que la tecnología, la dinámica del mercado y el uso social tienden a combinarse en diferentes modos y grados de intensidad.

Una de las materializaciones de dicha situación es la existencia de cuatro agentes estatales diferentes (INCAA, AFSCA, MINPLAN, AFTIC) que trabajan paralelamente temas que podrían (si no deberían) ser pensados y gestionados de modo integral, y en relación con el sector privado comercial y sin fines de lucro.

El Cine es el único que exhibe el desarrollo de una política de Estado permanente, duradera, con efectos concretos vinculadosalosobjetivos buscados, rediseño en base a necesidades emergentes, diagnóstico y planificación. No por azar atravesó las crisis económicas y políticas del país sin perder sus rasgos. Y obtiene incluso beneficios del resto de los marcos regulatorios, como la integración del Fondo de Fomento, la cuota de pantalla en Televisión abierta que habilita la LSCA para films nacionales y los planes y programas de fomento gestionados en conjunto por INCAA y MINPLAN para generar contenidos a ofrecer en la TDA. No ha sido alterada en el período estudiado más que en algunas cuestiones marcadas. $Y$ su implementación ${ }^{18}$ resultó ampliada en términos de inversión y cumplimiento de planes, programas y proyectos entre 2007 y 2015.

Las otras medidas son más recientes, llevan poco tiempo desde su implementación y fueron desarrolladas en un contexto general de conflicto entre el gobierno y los principales actores del sistema de medios, fundamentalmente con el Grupo Clarín. Tanto la LSCA como le decreto de adopción de TDA se dieron en un escenario conflictivo. Mientras que eso no se constata para la sanción de Argentina Digital, donde los principales críticos resultaron las empresas pymes y cooperativas. A su vez, en torno a la LSCA, los principales actores económicos resistieron las medidas impulsadas, con relativo éxito ${ }^{19}$.

Por todo esto, puede sostenerse que las regulaciones aprobadas en el período estudiado no responden a un conjunto integral y planificado de medidas, sino que incluso en algunas cuestiones se contradicen en sus formas: cambios menores en torno a una norma que establece la política pública más

18 Se recuerda que no es objeto de este trabajo, pero su evaluación puede ampliarse en Marino (2015).

19 Ver más en Di Santi y Riera (2015). 
duradera en el sector (el Cine); una ley con proceso democrático en su generación y aprobación (LSCA); un Decreto específico que asigna un rol central para el Estado (TDA); una ley propuesta por el PEN, sin indicios para la participación ciudadana y con aspectos por definir en su reglamentación (Argentina Digital).

\section{Palabras finales}

El recorrido propuesto en este trabajo implicó sistematizar las decisiones regulatorias de los sectores que hacen al EAA durante los dos mandatos de la presidenta Cristina Fernández de Kirchner, entre 2007 y 2015. Y permitió constatar las premisas propuestas.

El conjunto de avances tecnológicos de los últimos años transformó el sistema de medios masivos (prensa gráfica, radio y Televisión) generó nuevos medios (internet como medio y soporte, redes sociales, entre otros) y nuevas prácticas y consumos. Los cambios pueden ser clasificados en tres tipos: tecnológicos, económicos y políticos (de regulación). Cada uno de ellos impacta de diferente modo en el EAA.

Las formas de producir, distribuir contenidos en el espacio audiovisual cambia. Se adaptan a las nuevas formas de recepción y consumo en su estructura, pero también en sus contenidos. La constante mutación sucede en un marco de duda sobre los modelos de negocio tanto como sobre el futuro rol de los grandes medios y su relación con los usuarios-receptores-consumidores. Pero la mayor incertidumbre se posa sobre cuál será el mejor modo de articular desde el Estado políticas públicas que garanticen el acceso y la participación ciudadano de modo articulado y pertinente.

\section{Referencias}

BECERRA, M. Sociedad de la Información: proyecto, convergencia, divergencia. Buenos Aires: Grupo Ed. Norma, 2003.

BECERRA, M. De la concentración a la convergencia. Políticas de medios en Argentina y América Latina. Buenos Aires: Paidós, 2015.

DI SANTI, M y RIERA, A. Un mapa poco adecuado, en Marino, S.: El Audiovisual Ampliado. Políticas públicas, innovaciones del mercado y tensiones regulatorias en la industria de la televisión argentina frente a la convergencia. Buenos Aires: Universidad Del Salvador (en prensa), 2015. 
FREEDMAN, D. Las dinámicas del poder en la elaboración de políticas de medios en la actualidad. Media, Culture \& Society. Londres, v. 26, n. 6, 2006.

GETINO, O. Cine Argentino, entre lo posible y lo deseable. Buenos Aires: Ediciones Ciccus, 2005.

KRAKOWIAK, F.; MASTRINI, G.; Becerra, M.; Bizberge, A. El Estado como protagonista del desarrollo de la TDT en Argentina. Cuadernos de la Información, n. 31, Pontificia Universidad Católica de Chile, 2012.

MARINO, S. Industria cinematográfica latinoamericana: políticas públicas y su impacto en un mercado digital. Argentina. In Guillermo Mastrini y Marta Fuertes. Industria cinematográfica latinoamericana: políticas públicas y su impacto en un mercado digital. Buenos Aires: La Crujía, p. 67 - 110, abril 2014, 2014 a.

MARINO, S. Vaivén: desgranar moralejas en la Argentina de la ley audiovisual. In: Dossier $n^{\circ} 14$ del Observatorio Latinoamericano del Instituto de Estudios de America Latina y el Caribe (IEALC). Facultad de Ciencias Sociales, UBA, 2014 b.

MARINO, S. El Audiovisual Ampliado. Políticas públicas, innovaciones del mercado y tensiones regulatorias en la industria de la televisión argentina frente a la convergencia. Buenos Aires: Universidad Del Salvador (en prensa), 2015.

MARTEL, F. Cultura Mainstream: como nacen los fenómenos de masas. Madrid: Taurus, 2011.

MASTRINI, G. Las industrias culturales en Argentina: Economía y política del sector audiovisual en la década del ' $\mathbf{9 0}$. Tesis Doctoral, Universidad Complutense de Madrid, Facultad de Ciencias de la Información, Departamento de Periodismo III. Madrid, (mimeo), 2013.

MASTRINI, G. y BECERRA, Nuevas reglas de juego en telecomunicaciones en la Argentina, 2015. Disponible en: <http://observacom.org/nuevas-reglas-dejuego-en-telecomunicaciones-en-la-argentina/.> Acceso en: 10 set. 2015.

. Periodistas y magnates. Estructura y concentración de las industrias culturales. Buenos Aires: Prometeo, 2006.

; Marino, S; Gadano, J; Bieda, T y otros. Uso y consumo de la televisión digital en la Argentina. Maestría en Industrias Culturales de la Universidad Nacional de Quilmes, Quilmes, 90 pp, 2014. Disponible en:<http://es.scribd.com/ doc/238478587/Informe-TDA-Para-Web>. Acceso en: 5 set. 2015.

MONZONCILLO, J. M. Las nuevas televisiones: personalización e individualización, en La televisión etiquetada. Nuevas audiencias y nuevos negocios. Madrid: Planeta, 2011. 
OSZLAK, Oscar y O’DONNEL, Guillermo, Estado y Políticas Estatales en América Latina en Para Investigar la Administración Pública, INAP, Alcalá de Henares, 1984.

PAEZ, Triviño M. VOD la televisión sin programación y multipantalla. Aproximación al estudio del video bajo demanda en Argentina. Ponencia presentada al XXII Encuentro Nacional de Carreras de Comunicación (ENACOM), Mendoza, (e-book en diseño), 2014.

PEREA, R. y RETEGUI, L. Telecomunicaciones: Acceso, políticas y mercado. El caso de la telefonía móvil en Argentina, ponencia al PRIMER ENCUENTRO DE EQUIPOS DE INVESTIGACIÓN CEPOS / UNQ Perspectivas actuales de la Economía Política de la Comunicación, 2012.

PERELMAN, P y SEIVACH, P. La industria cinematográfica en la Argentina: Entre los límites del mercado y el fomento estatal, 2003. Disponible en: < $\underline{\text { http:// }}$ www.buenosaires.gob.ar/areas/hacienda/sis estadistico/ind cine arg2.pdf $>$. Acceso en: 10 set. 2015.

ROVITO, P. El nuevo paradigma del negocio cinematográfico, 2010. In: Valor y símbolo, dos siglos de industrias culturales en la Argentina. Buenos Aires: Secretaría de Cultura. Sistema de Información Cultural de la Argentina (SINCA), p. 105-112, 2010.

SAHORES, Esteban. Entrevista realizada con el periodista especializado Esteban Sahores. MARINO, Santiago. Buenos Aires, mayo de 2014.

VAN CUILENBURG, J y MCQUAIL, D. Cambios en el paradigma de política de medios. Hacia un nuevo paradigma de políticas de comunicación. In: European Journal of Communication, v. 18. n. 2. Londres: Sage, p. 181-207, 2003. 